\title{
Determination of physic and mechanical parameters of high-density polyethylene based on relaxation curves due to the presence of hydroxyapatite and ionizing radiation
}

\author{
Stepan Litvinov ${ }^{1, *}$, Serdar Yazyev ${ }^{1}$, and Dmitriy Vysokovsky ${ }^{1}$ \\ ${ }^{1}$ Don State Technical University, Rostov-on-Don, Russia
}

\begin{abstract}
Relatively low physical and mechanical parameters of polymers negatively affect the possibility of using this material everywhere. There are ways to improve the elastic and rheological characteristics of some varieties of polymers: addition of additives and irradiation with ionizing radiation. However, these methods can lead both to the cross-linking of polymer molecules with the improvement of its parameters, and to its destruction. In this article shown the theoretical definition of physic and mechanical parameters of high-density polyethylene by known stress relaxation curves, and a comparison of the experimental data of highdensity polyethylene (HDPE) with the data obtained theoretically, which, in turn, saves material resources and man-hours for the experiment and its subsequent analysis.
\end{abstract}

\section{Conduct}

High-density polyethylene is a very common artificial material. It is used in food, tube, construction and other industries, but he also took a special place in medicine in the manufacture of implants and prostheses. This is due to his biocompatibility, its characteristics coincide with the characteristics of the natural bone [1]. This property is very important, since it eliminates the possibility of osteoporosis in the operation of the prosthesis. However, rheology, low modulus of elasticity and low biological activity do not always allow the use of this polymer.

In works [2-3] attempts were made to improve the above properties, including the addition of various impurities to the basic composition of HDPE. One of these additives may be hydroxyapatite, which has a positive effect on the stiffness of the composite and biological activity. The strength of HDPE is provided by itself. The viscoelastic behavior of the material is easily described in time and allows us to make a prediction about the longterm strength [4-6]. Thus, the use of hydroxyapatite (hereinafter - HA) as a reinforcing element of the polymer material has a beneficial effect on the biological activity and rheological behavior of HDPE.

\footnotetext{
Corresponding author: litvstep@gmail.com
} 
Also, favorable transformations for polymeric material can be caused by ionizing radiation. As the effect of gamma radiation, molecules can both collapse and become larger. As is known, one of the main characteristic features of a polymer is the size of its molecule. In 1953, Charlsby and Lawton proposed a classification of polymers depending on their relationship to ionizing radiation: structuring, in which transverse bonds are formatted, that is, gamma radiation favorably affects the process of cross-linking the molecule of the composite, and destructuring, in which destruction occurs (rupture of molecules).

Naturally, the crosslinking process contributes to a change in the properties of the polymer. First, it affects the solubility of the polymer. A large number of types of solvents can dissolve the non-cross-linked polymer due to the relatively easy disconnection of polymer chains by solvent molecules. However, the process becomes more difficult with increasing number of crosslinks in the polymer molecule, at the same time it ceases from a certain moment determined by the doses of ionizing radiation, depending on the molecular weight of the polymer.

Secondly, the susceptibility of high temperatures to a polymer changes. Thus, at temperatures above $105-115^{\circ} \mathrm{C}$, the unirradiated material is a viscous liquid. But after irradiation, the polymer acquires the property of rubber due to the crosslinking of its molecules. If a tensile load is applied to the product from the irradiated polymer, the behavior of the sample becomes like a spring. Instead of spreading the polymer, there is a lengthening of the product, as there is a straightening of the individual units of the molecule grid. And after removing the load, the former form of the grid is completely restored. However, in the case of significant thickness grid behavior of the polymer under the influence of an external load may deteriorate, since the possibility of individual units to straighten eliminates. Then, even with a relatively small mechanical loading, the product loses its elasticity and ability to stretch, becomes brittle.

Thus, at the present time, gamma radiation is used in practice. So the products made of polyethylene, whose task is to work in conditions of elevated temperatures, are preliminarily irradiated. In 1959, in the United States, one company produced 400 tons of irradiated polyethylene film. As a result, at low cost, compared with a film of conventional polyethylene, it was possible to achieve an increase in the film strength by a factor of 5 , and the ability to stretch it by a factor of 2 . At the same time, such products are capable of operating under temperature conditions up to $250{ }^{\circ} \mathrm{C}$. V.L. Karpov and his co-workers suggested using irradiated polyethylene for the production of insulation of wires operated at elevated temperatures. If we compare irradiated polyethylene and unirradiated in ordinary temperatures, the cross-linked polymer, compared to conventional, has an increased mechanical strength.

\section{Experimental procedure}

Based on the results of experimental data of the work of medical engineers [7], an analysis was made of the change in the elastic and rheological parameters of HDPE with the addition of $\mathrm{HA}$ and the irradiation of the material to $70 \mathrm{kGy}$ (the irradiation power was increased by $5 \mathrm{kGy}$ every hour).

Experimental studies on the relaxation of the stresses of pure HDPE and its nanocomposite samples were carried out at a constant temperature $T=25{ }^{\circ} \mathrm{C}$ and with a constant deformation of the $\operatorname{rod} \varepsilon=3 \%$, while a decrease in the stress level during 3 hours.

The results of the stress relaxation data experienced irradiated and unirradiated HDPE and nanocomposites containing 30\% HA shown in figure 1 . An analysis of the obtained curves shows that the relaxation of the stresses of nanocomposites with HA and irradiated samples is more pronounced than in pure HDPE samples. In addition, the level of initial 
stress and stress at the end of the relaxation process have higher values of nanocomposite samples with HA than samples from pure HDPE. The growth of the voltage at the initial instant of time is explained by the presence of HA particles in the polymer matrix of HDPE and, as a consequence, a change in the stiffness. Also the analysis of figure 1 shows that the relaxation of the stress of HDPE with HA additives and irradiation after 3 hours of testing was $37 \%$ of its value at the initial time, while for the sample without additives and irradiation it was only $24 \%$ of the initial value. Nanocomposite with HA is able to reduce stresses in bone substitutes, which positively affects its work together with the body during prosthetics.

To realize the idea, which is mentioned in the annotation to this article, it is necessary to define elastic and rheological constants of HDPE in theory. To do this, the rate of change of the stress function over time is first determined. You can use the function polyfit from the program complex Matlab, which has the form $p=\operatorname{polyfit}(x, y, n)$. It finds the coefficients of the polynomial $p(x)$ of $\mathrm{n}$ degree, approximating the function $y(x)$ using the least squares method. The output is a string $\mathrm{p}$ of length $n+1$, containing the coefficients of the approximating polynomial:

$$
p(x)=p_{1} x^{n}+p_{2} x^{n-1}+\ldots+p_{n} x+p_{n+1}
$$

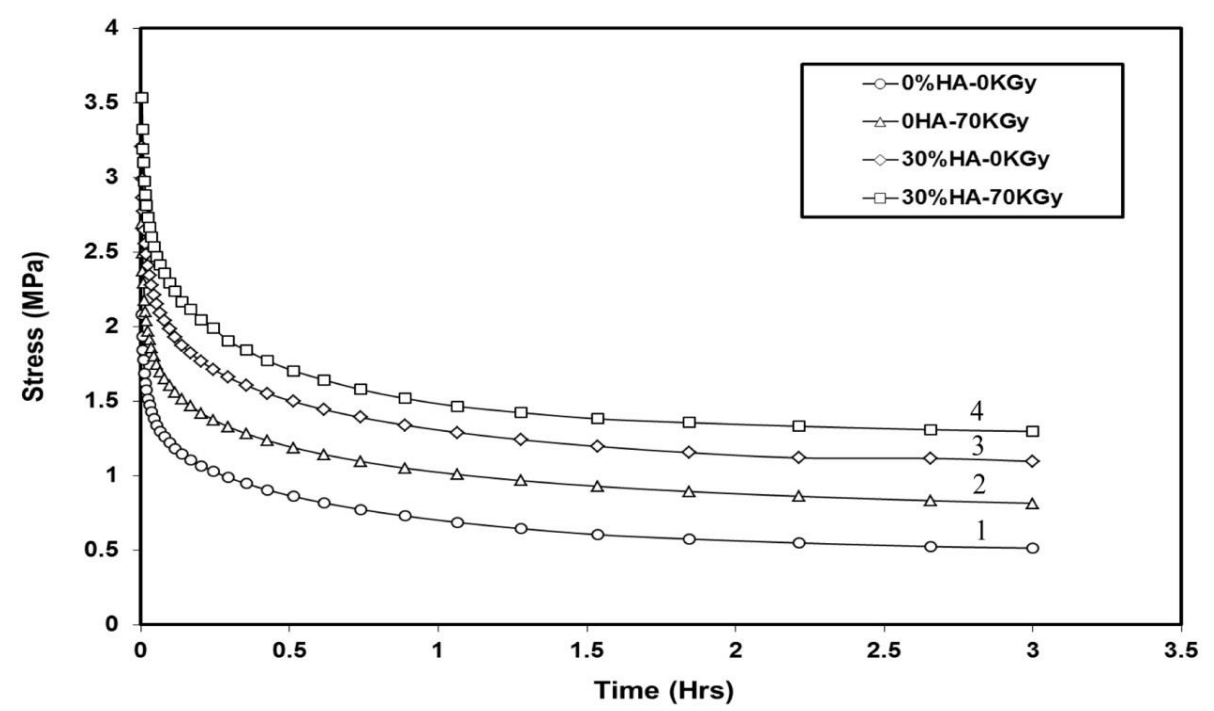

Fig. 1. Results of stress relaxation of HDPE: 1 - pure HDPE, without irradiation; 2 - pure HDPE, irradiation of $70 \mathrm{kGy} ; 3$ - HDPE $+30 \% \mathrm{HA}$, without irradiation; 4 - HDPE $+30 \% \mathrm{HA}$, irradiation of $70 \mathrm{kGy}$.

The selection of the coefficients of the approximating polynomial occurs on three points (a square polynomial or a polynomial of degree 2), which with taking into account the initial functions has the form:

$$
\sigma_{i}=p_{1} t^{2}+p_{2} t+p_{3}
$$

To determine the desired derivative of the function at each point, it is necessary to differentiate expression (2) in time. The dash « » denotes the time derivative:

$$
\sigma_{i}^{\prime}=2 p_{1} t+p_{2}
$$

Thus, using the function polyfit allows you to quickly and accurately obtain the necessary coefficients $p_{1}$ and $p_{2}$. After this, it becomes possible to determine the growth 
rate of the stress function in time, for this it is necessary to use expression (3), and then finding the elastic and rheological parameters of the nonlinear Maxwell-Gurevich equation. The results are shown in Table 1 .

Table 1. Elastic and rheological parameters of HDPE.

\begin{tabular}{|c|c|c|c|c|}
\hline \multicolumn{1}{|c|}{ HDPE } & $E, M P a$ & $E_{\infty}, M P a$ & $m^{*}, M P a$ & $\eta_{0}^{*}, M P a \cdot h$ \\
\hline $0 \%$ HA, 0 kGy & 693,9890 & 228,8515 & 5,5445 & 1113,0 \\
\hline $0 \%$ HA, 70 kGy & 897,5469 & 388,1827 & 6,4429 & 1734,4 \\
\hline 30\% HA, 0 kGy & 1069,3 & 556,7567 & 8,0948 & 1832,5 \\
\hline 30\% HA, 70 kGy & 1178,4 & 684,5894 & 10,1390 & 1768,4 \\
\hline
\end{tabular}

As a result, 4 values of each variable were obtained depending on the fraction of HA and the level of irradiation of the polymer $F$, to determine the variables at intermediate values of the fraction of the HA and the irradiation level $F$, we interpolate by means of a polynomial of the form:

$$
f(x, y)=a+b \times x+c \times y+b \times x \times y
$$

Then the elastic modulus expression, depending on the fraction of the HA and the irradiation level $F$, has the form:

$$
E(H A, F)=694+1251 \times H A+2,908 \times F-4,498 \times H A \times F \quad[M P a]
$$

The expression of the modulus of elasticity, depending on the fraction of HA and the level of irradiation $F$ :

$$
E_{\infty}(H \mathrm{~A}, \Phi)=228,9+1093 \times H A+2,276 \times F-1,5 \times H A \times F \quad[M P a]
$$

The expression of the velocity modulus as a function of the fraction of the HA and the irradiation level $F$ :

$$
m^{*}(H A, F)=5,545+8,501 \times H A+0,01283 \times F+0,05456 \times H A \times F \quad[M P a]
$$

The expression for the coefficient of initial relaxation viscosity as a function of the fraction of HA and the level of irradiation $F$ :

$$
\eta_{0}^{*}(H A, F)=1113+2398 \times H A+8,877 \times F-32,64 \times H A \times F \quad[M P a \times h]
$$

The analysis of expressions (5) - (8) shows that with an increase in the fraction of HA and the irradiation level $F$ all elastic and rheological parameters increase. The exception is the coefficient of the initial relaxation viscosity, which, when the HA is simultaneously injected and the material is irradiated, is approximately the same as when injecting HA or only irradiating HDPE.

\section{Results and discussion}

In order to verify the correctness of the determination of the elastic and rheological parameters of the Maxwell-Gurevich equation, it is first necessary to obtain the theoretical stress relaxation curves using the obtained dependences (5) - (8), and then compare them with the experimental data for which they were determined (figure 1).

It is known that the total deformation of the test rod is composed of elastic and highly elastic, and is equal to a certain constant value. Since in this problem a uniaxial stress state is observed, in the subsequent calculations the index of the axis of the $\operatorname{rod} x$ is not necessarily used.

$$
\varepsilon=\varepsilon_{e l}+\varepsilon_{c r}=\frac{\sigma}{E}+\varepsilon_{c r}=\text { const }=0,03 \mathrm{~mm}
$$

From expression (9) it is possible to determine the stress at any time. 


$$
\sigma=E\left(\varepsilon-\varepsilon_{c r}\right)
$$

The stress at the initial instant of time is determined by multiplying the modulus of elasticity of the material by the initial deformation, constant throughout the calculation time.

$$
\sigma(0)=E \times \varepsilon
$$

At the initial time, the high-elastic deformation is zero $\left(\varepsilon_{c r}=0\right)$, Thus, it is possible to determine the creep strain in the following time step:

$$
\begin{aligned}
& \varepsilon_{c r}(\mathrm{t}+1)=\varepsilon_{c r}(\mathrm{t})+\frac{\partial \varepsilon_{c r}(\mathrm{t})}{\partial t} \Delta t ; \\
& \frac{\partial \varepsilon_{c r}(\mathrm{t})}{\partial t}=\frac{f^{*}}{\eta^{*}} ; \quad f^{*}=\sigma-E_{\infty} \varepsilon_{c r} ; \quad \eta^{*}=\eta_{0}^{*} \exp \left(-\frac{\left|f^{*}\right|}{m^{*}}\right)
\end{aligned}
$$

Considering the foregoing, the stress function $f^{*}$ is determined by the following formula:

$$
f^{*}=\sigma-E_{\infty} \times \varepsilon_{c r}=\sigma
$$

Thus, to determine the high-elastic deformation in the next time interval $\varepsilon_{c r}(t+1)$ all terms are known. It also becomes possible to determine the stresses at the next time step $\sigma(t+1)$ with the help of expression (10). This process is repeated until the last calculation time point is reached.

To estimate the reliability of the obtained equations $(5)-(8)$, in figure 2 compares the experimental data of stress relaxation (dashed lines) with theoretical (solid lines) obtained from expressions (5) - (8). The coincidence of experimental and theoretical lines is very good, and small discrepancies are explained by the inaccuracy of processing data obtained from an analysis of the stress relaxation curves.

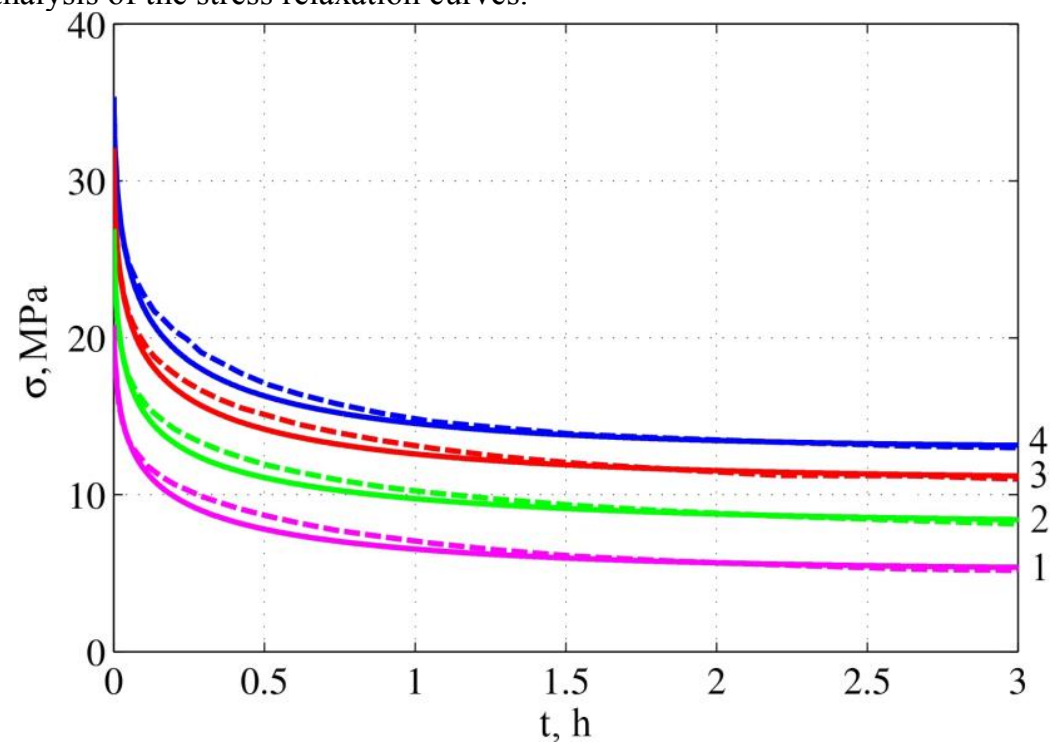

Fig. 2. Results of comparison of experimental curves (dashed lines) with theoretical curves (solid lines): 1 - pure HDPE, without irradiation; 2 - pure HDPE, irradiation of $70 \mathrm{kGy} ; 3$ - HDPE + $30 \% \mathrm{HA}$, without irradiation; 4 - HDPE $+30 \% \mathrm{HA}$, irradiation of $70 \mathrm{kGy}$ 


\section{Conclusions}

Thus, it becomes possible, when analyzing the experimental stress relaxation data, to predict the properties of polymer materials at intermediate values of the parameters: the proportion of hydroxyapatite and the level of radiation. Undoubtedly, this opportunity leads to a significant saving of material resources and man-hours for the experiment and its subsequent analysis.

\section{References}

1. J. Nagels, M. Stokdijk, P.M. Rozing, Journal of shoulder and elbow surgery, 12, 35-39 (2003)

2. K. Li, S.C.Tjong, Journal of Macromolecular Science, 50, 1325-1337 (2011)

3. K.E. Tanner, R.N. Downes, W. Bonfield, British Ceramic Transactions, 93, 104-107 (1994)

4. H. Fouad, Materials and Design, 32, 1974-1980 (2011)

5. H. Fouad, R. Elleithy, Journal of the mechanical behavior of Biomedical materials, 4 , 1376-1383 (2011)

6. S.V. Litvinov, L.I. Trush, A.A. Avakov, International Conference on Industrial Engineering, Applications and Manufacturing (ICIEAM), 1-5 (2017)

7. O.Y. Alothman, Biomedical engineering online, 13 (2014) 